\title{
Bildgebende MR-Verfahren zur Erfassung von Körperfunktionen
}

Bösiger, $\mathrm{P}$

DOI: https://doi.org/10.1515/bmte.1995.40.s2.16

Posted at the Zurich Open Repository and Archive, University of Zurich ZORA URL: https://doi.org/10.5167/uzh-155418

Journal Article

Published Version

Originally published at:

Bösiger, P (1995). Bildgebende MR-Verfahren zur Erfassung von Körperfunktionen. Biomedizinische Technik. Biomedical engineering, 40(s2):16-19.

DOI: https://doi.org/10.1515/bmte.1995.40.s2.16 


\title{
Bildgebende MR-Verfahren zur Erfassung von Körperfunktionen
}

\section{P. BÖSIGER}

\author{
Institut für Biomedizinische Technik, Universität und ETH Zürich
}

Die Magnetresonanz-Tomographie, wie sie heute in der klinischen Diagnostik eingesetzt wird, erzeugt anatomische Schichtbilder des menschlichen Körpers, die eine hohe Differenzierung der Weichteilstrukturen erlauben. Neue bildgebende spektroskopische Magnetresonanzverfahren eröffnen aber auch neue Wege zur nichtinvasiven Analyse von funktionellen Eigenschaften des menschlichen Körpers und seiner Organe. In unseren Forschungsprojekten werden neue Untersuchungstechniken zur Erfassung von Funktionen des Herz-Kreislaufsystems (Herzbewegung, Blutfluss), des Magens (Motilität, Nahrungsverarbeitung), des Gehirns (Stoffwechsel) und weiterer Organe entwickelt und in Zusammenarbeit mit einschlägigen Spezialisten des Universitätsspitals Zürich und anderer internationaler Fachgruppen in medizinischen Studien eingesetzt.

\section{Bildgebende Magnetresonanzverfahren zur} Analyse von Herz- und Kreislauf-Funktionen

Zur Analyse der Bewegung des Herzens wird die neue Technik des Myocardial Taggings entwickelt. Dabei wird enddiastolisch durch räumliche Modulierung der Magnetisierung des Herzmuskelgewebes ein muskelfestes Gittermuster über das Herz gelegt $(1,2)$. Aus den Verschiebungen und den Verzerrungen dieses Gitters in Zeitreihen von Bildern, die anschliessend während des Herzzyklus aufgenommen werden, lassen sich charakteristische Bewegungsmuster des gesunden Herzens und bei verschiedenen Erkrankungen ableiten. Aufgrund der Ergebnisse von Modellrechnungen zum Kontrast zwischen dem Gitter und dem nicht markierten Gewebe wurden neue Messtechniken entwickelt, welche eine signifikante Verbesserungen des Kontrastes zwischen dem Gitter und dem Gewebe erbrachten. Damit wurde eine Bewegungs- analyse über den gesamten Bewegungszyklus des Herzen unter gleichzeitiger Elimination von Bewegungseffekten senkrecht zur abgebildeten Schicht möglich $(3,4)$ (Bild 1).

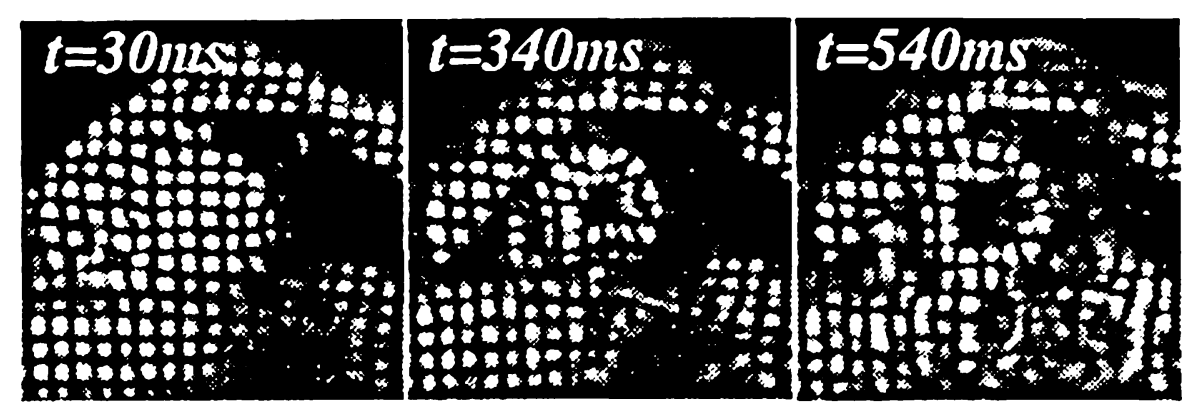

Bild 1: Schichtbilder des Herzens mit aufmoduliertem Gittermuster zu drei verschiedenen Zeitpunkten des Herzzyklus. Von links nach rechts: $t=30$ ms nach der $R$-Zacke des EKG (Frühe Systole); $t=340 \mathrm{~ms}$ (End Systole); $t=540 \mathrm{~ms}$ (Späte Diastole).

Zur Auswertung der Bildsequenzen wurde eine computerunterstützte Prozedur entwickelt, welche in einem ersten Schritt die Gittermuster auf den Bildern identifiziert. Anschliessend werden die Veränderungen der Lage der Kreuzungspunkte des Gitters vom einen zum jeweils nächsten Bild durch die gesamte Sequenz bestimmt
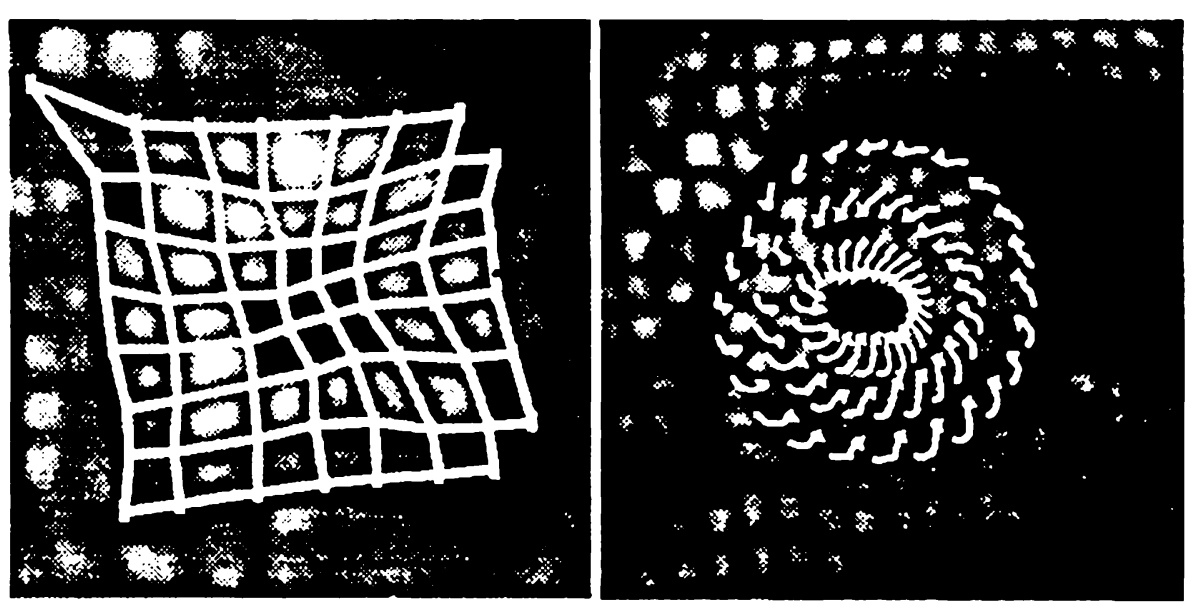

Bild 2: Bewegungsmuster der linken Kammer an der Herzspitze. Links: Deformiertes Gitter am Ende der Kontraktionsphase (=Systole). Rechts: Lokale Bewegung während der Systole dargestellt mit Tajektorien. Neben der Bewegung des Gewebes in Richtung des Kammerzentrums sind deutlich Drehbewegungen der Herzspitze im Gegenuhrzeigersinn zu beobachten.

und Bilder errechnet, auf denen die lokalen Bewegungsvektoren für verschiedene Schichten während des gesamten Herzzykluses dargestellt sind (5) (Bild 2).

Erste Ergebnisse einer Studie, die in Zusammenarbeit mit der Abteilung für Kardiologie (Prof. 
Dr. O.M. Hess) des Universitätsspitals Zürich durchgeführt wurde, zeigen beim gesunden Herzen für basale Schichten neben der Bewegung des Muskelgewebes in der Richtung des Kammerzentrums eine leichte Drehbewegung im Uhrzeigersinn (6). Bei Gewebe an der Herzspitze tritt eine erhebliche Drehung im Gegenuhrzeigersinn auf. Es findet also gleichsam ein Auswringen des Blutes aus dem Herzen statt. Bei Patienten mit Hypertrophien infolge von Aortenstenosen ist der zeitliche Ablauf der Bewegung stark verändert, während die Bewegungen bei Ruderern mit physiologischen Hypertrophien wie bei Gesunden ablaufen. Bei Herzinfarkten liegen sowohl bei der Kontraktion (systolische Herzphase) als auch bei der anschliessenden Entspannung des Muskels (diastolische Phase) vor allem im Bereich des Infarktes, aber auch global massiv veränderte Bewegungsmuster vor.

Bildgebende Magnetresonanz-Verfahren erlauben im weiteren die angiographische Darstellung von Gefässstrukturen und die Erfassung der Blutgeschwindigkeitsverteilungen in Gefässen des menschlichen Körpers. Zur Analyse des Blutflusses werden die Phasenverschiebungen der Magnetresonanzsignale ausgewertet, welche in erster Näherung proportional zur lokalen Blutflussgeschwindigkeit sind $(7,8)$. Speziell

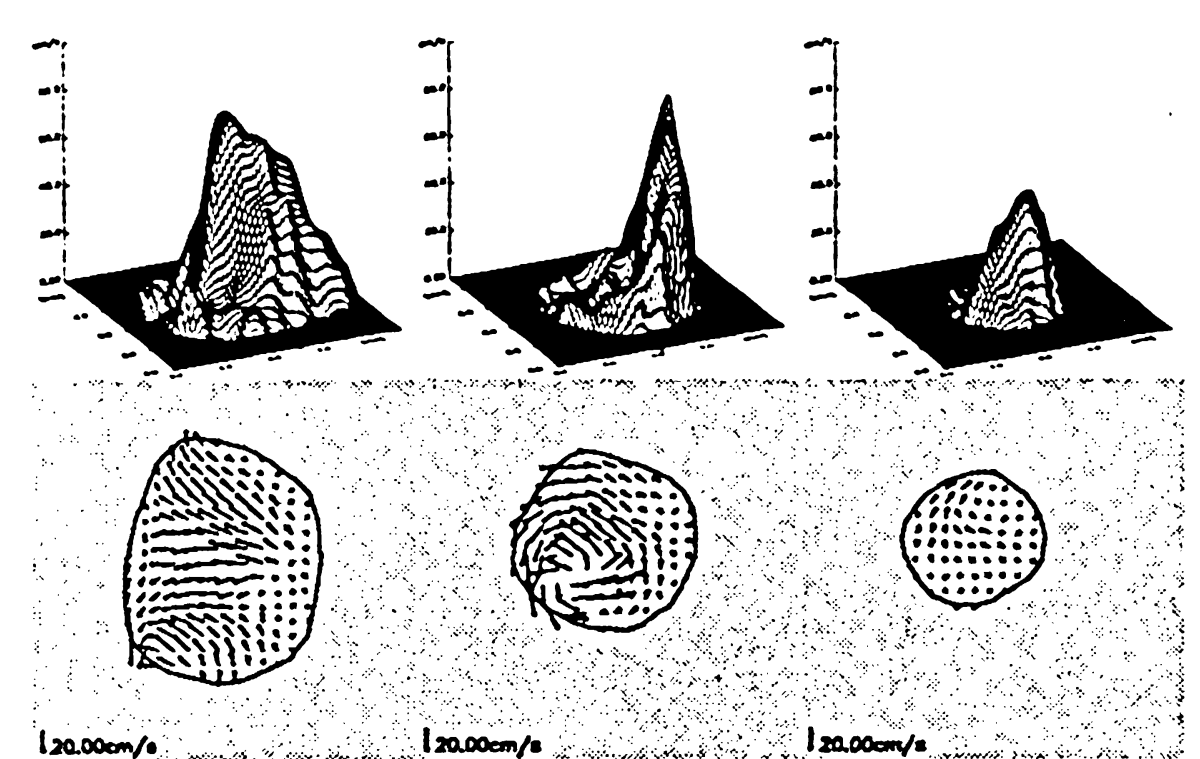

Bild 3: Obere Reihe von links nach rechts: Axiale Blutgeschwindigkeitsprofile in der Carotis Communis, Interna und Externa. Untere Reihe: Entsprechende Blutflussgeschwindigkeiten in transversaler Richtung.

entwickelte Techniken wie FAcE (free induction decay acquired echos) (9) oder Half-EchoSampling zeichnen sich durch extrem kurze Echozeiten und damit durch hohe Unterdrükkung von flussinduzierten Bildartefakten aus. Sie ermöglicht die sehr detaillierte. Erfassung der über eine gewisse Anzahl von Herzzyklen gemittelten Geschwindigkeitsprofile auch in Gefässstrukturen mit komplizierten Flussverhältnissen, wie sie bei Gefässverzweigungen, bei Stenosen und Aneurysmen vorliegen, und in Gefässen innerhalb des Schädels und des Beckens (10). Bild 3 zeigt die Ergebnisse von Flussmessungen an der Carotisbifurkation eines gesunden Probanden. Deutlich sind die teilweise sehr komplizierten Geschwindigkeitsprofile im Bifurkationsbereich erkennbar. Der kurzzeitige Rückfluss, die sogenannte dichrotische Welle, ist typisch für gesunde Probanden im Ruhezustand und wird durch die teilweise Reflexion des Flusspulses in peripheren Gefässen mit veränderter Gefässimpedanz erzeugt. Gegenwärtig sind Weiterentwicklungen dieser Flussmesstechnik im Gange, die auch Messungen des Flusses in Koronararterien ermöglichen werden. Für die Gefässangiographie wird die Tatsache ausgenützt, dass bei gewissen Messtechniken infolge der repetitiven Anregung der bei der Bilderzeugung beobachteten Spins der Wasserstoffkerne einer Schicht eine teilweise Sättigung auftritt. Dadurch nimmt die Signalintensität des stationären Gewebes ab; das Gewebe crscheint dunkel im Bild. Liegen Blutgefässe in dieser Schicht, wird das gesättigte Blut zwischen den Anregungen durch neu in die Schicht einfliessendes ungesättigtes Blut ersetzt, welches starke Kernresonanzsignale erzeugt. Die Blutgefässe erscheinen deshalb als helle Strukturen. Aus Stapeln von Schichtbildern lassen sie sich segmentieren und als dreidimensionale Gebilde darstellen. Für die Darstellung der Herzkranzgefässe ist eine Synchronisation der Messzyklen sowohl auf das EKG als auch auf die Atembewegung des Patienten notwendig (11). Bild 4 zeigt links die dreidimensionale angiographische Rekonstruktion der grossen Herzkranzgefässe eines Patienten mit einem Verschluss der linken Koronararterie, wie er nach starken Herzbeschwerden diagnostiziert wurde.

Rechts ist die Situation nach Erweiterung mit einem Ballonkatheter gezeigt, nach der sich wieder normaler Blutfluss eingestellt hat. $\mathrm{Da}$ die Magnetresonanz-Angiographie völlig nichtinvasiv ist und dabei keine den Organismus belastende Kontrastmittel und keine ionisierende Strahlung eingesetzt wird, eignet sie sich vor- 


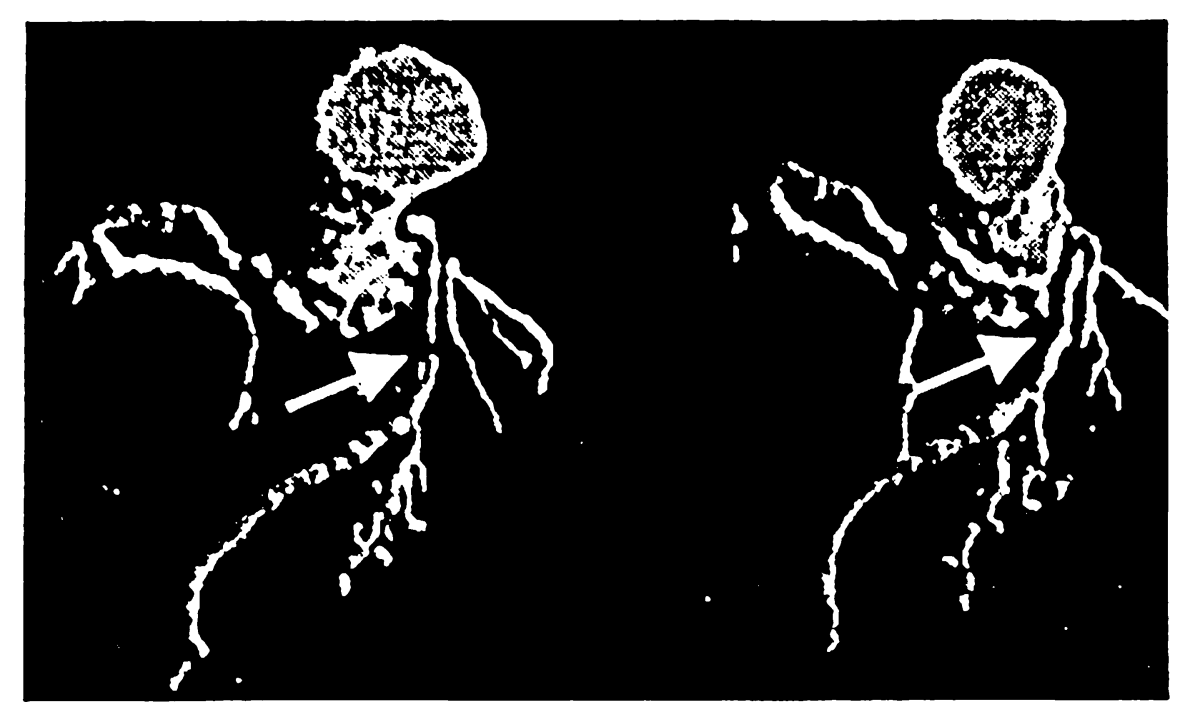

Bild 4: Magnetresonanz-Angiogramme der grossen Koronararterien, wie sie heute ohne Kontrastmittel und ohne ionisierende Strahlung aufgenommen werden können. Das Bild links zeigt einen Verschluss der linken Koronararterie (Pfeil), der im rechten Bild nach der Dilatation des Gefässes mit einem Ballonkatheter verschwunden ist.

züglich für periodische Nachkontrollen der Durchgängigkeit behandelter Gefässe. Sie wird gegenwärtig ebenfalls in Zusammenarbeit mit der Abteilung für Kardiologie in einer klinischen Studie zur Erkennung einer nach der Ballondilatation in gewissen Fällen auftretenden Restenosierung eingesetzt.

\section{Bildgebende Magnetresonanzverfahren Messung der Magenfunktionalität}

In der Gastroenterologie befasst man sich unter anderem mit der Untersuchung und der Erfassung der Magenfunktionalität. Das Wissen des zeitlichen Verlaufs der Magenentleerung nach Aufnahme einer Testmahlzeit und der Motilität d.h. der Kontraktionen der Magenwand, welche verantwortlich sind für die Zerkleinerung und den Weitertransport der Nahrung, hilft, die Vorgänge bei der Nahrungsverarbeitung zu verstehen. Im Weiteren liefert es wertvolle Beiträge zur Diagnose und zur Erfassung des Therapieverlaufes bei gewissen Magenerkrankungen, wie zum Beispiel der Gastroparesis bei Diabetikern. Im Gegensatz zu anderen verwendeten Methoden (Szintigraphie, Manometrie, Ultraschall) erlaubt MRI eine nichtinvasive Untersuchung der Magenentleerung und der Magenmotilität, bei welcher der Patient auch keiner radioaktiven Belastung ausgesetzt wird. Dazu wird dem nüchternen Probanden vor der Untersuchung eine flüssige oder feste Testmahlzeit unter Zugabe eines Kontrastmittels verabreicht. Unmittelbar nach Aufnahme der Testmahlzeit wird die Untersuchung mit einer Messung des Volumens des Mageninhaltes begonnen. Zu diesem Zweck werden in einer transversalen Schichtorientierung mehrere Schichtbilder aufgenommen, welche den gesamten Magen überdecken. Bild 5 zeigt Bilder einer Volumenmessung, auf wel-

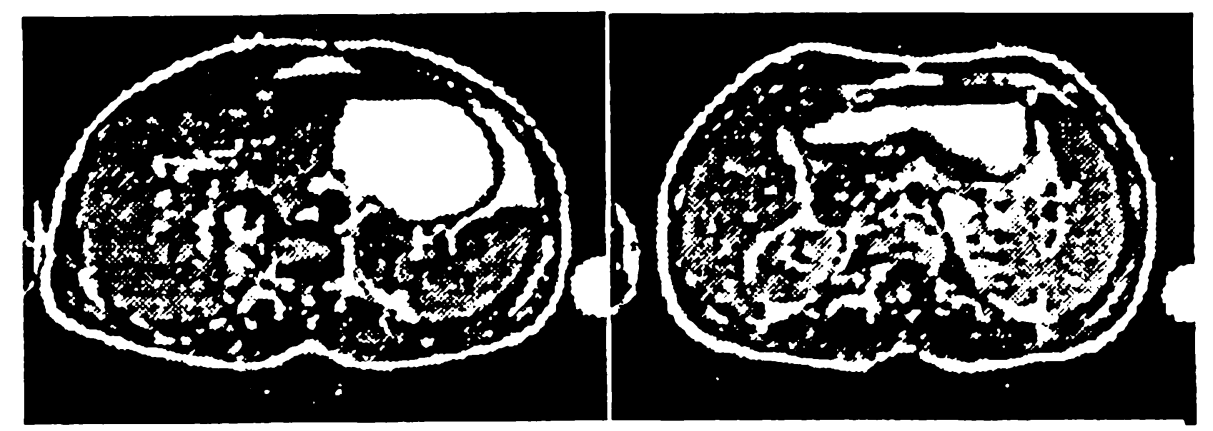

Bild 5: Zwei transversale Schichtbilder des Magens nach Verabreichung der Testmahlzeit wie sie zur Volumenmessung des Mageninhalts angefertigt werden. Der Mageninhalt ist als weisse Fläche deutlich zu erkennen.

chen der Mageninhalt wegen des Kontrastmittels deutlich als helle Fläche zu erkennen ist.

Die ebenfalls sehr helle runde Fläche ausserhalb des Körpers stammt von einer Referenz, welche neben dem Probanden liegend mitgemessen wird, um die Magensaftsekretion rechnerisch abschätzen zu können. Anschliessend an die Volumenmessung erfolgt eine Messung der Motilität. Die fortlaufenden im proximalen Magen ihren Anfang nehmenden und bis zum Pylorus verlaufenden Kontraktionen werden in einer koronalen Schichtorientierung mit einer dynamischen Sequenz von im Abstand von je einer Sekunde aufeinanderfolgenden Aufnahmen erfasst. Werden diese Bilder in einer Animation gezeigt, sind die sich ausbreitenden Kontraktionen deutlich zu erkennen Bild 6 zeigt eine Folge von derartigen Motilitätsbildern, auf denen die Ma-

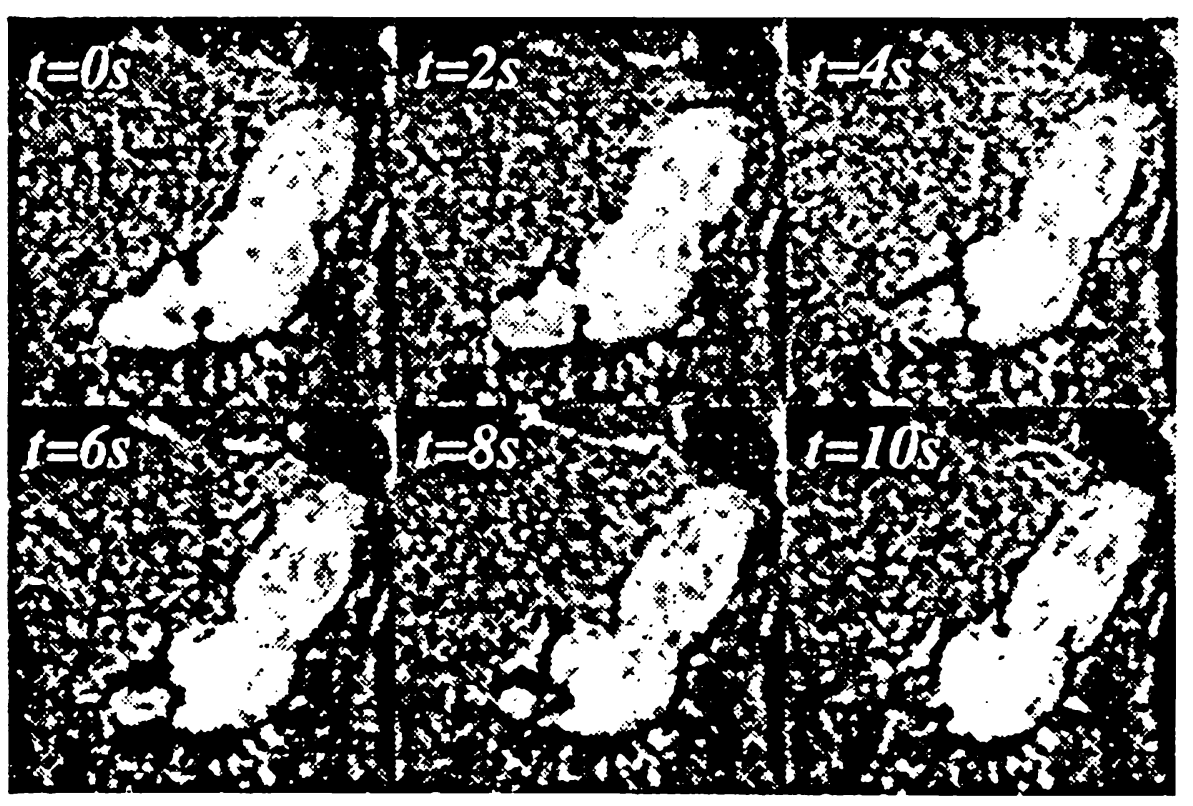

Bild 6: Sechs koronale Schichten einer dynamischen Messung zur Erfassung der Magenmotilität. Von Bild zu Bild leicht zu erkennen sind die gegen den Magenausgang laufenden Kontraktionen der Magenwand.

genwandkontraktionen ersichtlich sind. Die beiden Messungen werden ineinander verschachtelt während 120 Minuten alle 15 Minuten wiederholt um die Volumenabnahme des Mageninhal- 
tes während der Entleerungsphase zu beobachten und Zusammenhänge zwischen dem Entleerungsgrad des Magens und der Stärke der Motilität zu erkennen. Zur Auswertung der Bildsequenzen wurde eine Auswertungssoftware entwickelt, welche in einem ersten Schritt in den Entleerungs- und den Motilitätsbildern den Mageninhalt mit Hilfe eines Konturdetektionsalgorithmus segmentiert. Dies ist mit einem Algorithmus realisiert worden, der aufgrund des Helligkeitsunterschiedes zwischen dem Mageninhalt und dem umliegenden Gewebe den Mageninhalt segmentiert. Anschliessend wird durch dreidimensionale Rekonstruktion zu jedem Zeitpunkt das Volumen des Mageninhaltes berechnet, die daraus resultierende Entleerungskurve aufgezeichnet und die Halbwertszeit der Entleerung bestimmt. Ebenfalls ermittelt werden Motilitätsparameter wie Ausbreitungsgeschwindigkeit, Frequenz und Amplitude der Kontraktionen. Erste Ergebnisse von Studien, die in $\mathrm{Zu}-$ sammenarbeit mit der Abteilung für Gastroenterologie des Universitätsspitals Zürich durchgeführt wurden, zeigen, dass es mit dieser Methode möglich ist, den Einfluss verschiedener Testmahlzeiten oder die Wirkung von Medikamenten auf die Magenentleerung und die Motilität zu dokumentieren (12).

Der Autor dankt den Herren Dr. S.E. Fischer, Dr. D. Meier, Dr. M.B. Scheidegger, R. Botnar, G. Crelier, P. Kunz und M. Stuber für die Bearbeitung der Projekte und der Kommission zur Förderung der wissenschaftlichen Forschung und der Firma Philips Medical Systems für ihre Unterstützung. Er dankt Prof. Dr. O.M. Hess, Kardiologie, und Prof. Dr. M. Fried, Gastroenterologie des Universitätsspitals Zürich für die wissenschaftliche Zusammenarbeit.

\section{Literatur:}

1.) Zerhouni EA, Parish DM, Rogers WJ, Yang A, Saphiro EP. Human Heart: Tagging with MR Imaging - A Method for Noninvasive Assessment of Myocardial Motion. Radiology 169, 59-63, 1988.

2.) Axel L, Dougherty L. MR Imaging of Motion with Spatial Modulation of Magnetization. Radiology 171, 841-845, 1989.
3.) Fischer S.E., McKinnon G.C., Maier S.E., Boesiger P. Improved Myocardial Tagging Contrast. Magn Reson Med 30, 191-200, 1993.

4.) Fischer S.E., McKinnon G.C., Scheidegger M.B., Prins W., Meier D., Boesiger P. True Myocardial Motion Tracking. Magn Reson Med 31, 401-413, 1994.

5.) Maier S.E., Fischer S.E., McKinnon G., Hess O.M., Krayenbuehl H.P., Boesiger P. Acquisition and Evaluation of Tagged Magnetic Resonance Images of the Human Left Ventricle. Comput Med Imaging and Graphics 16, 73-80, 1992.

6.) Maier S.E., Fischer S.E., McKinnon G., Hess O.M., Krayenbuehl H.P., Boesiger P. Evaluation of Left Ventricular Segmental Wall Motion in Hypertrophic Cardiomyopathy with Myocardial Tagging. Circulation 86, 1919-1928, 1992.

7.) Van Dijk P. Direct cardiac NMR imaging ofheart wall and flow velocity. J Comput Assist Tomogr 8(3), 429-436, 1984.

8.) Meier D., Boesiger P., Maier S. Quantitative Flow Measurements on Phantoms and on Blood Vessels with MR. Magn Reson Med 8, 25-34, 1988.

9.) Scheidegger M.B., Maier S.E., Boesiger P. FID-Acquired Echoes (FAcE): A Short Echo Time Imaging Method for Flow Artefact Suppression. Magn Reson Imaging 9, 517-524, 1991.

10.) Boesiger P., Maier S.E., Liu K., Scheidegger M.B., Meier D. Visualization and Quantification of the Human Blood Flow by Magnetic Resonance Imaging. J Biomechanics 25, 55-67, 1992.

11.) Scheidegger M.B., Hess O.M. Vassalli G., Boesiger P. Magnetic Resonance Imaging of the Coronary Arteries. Technology and Health Care 2, 255-265, 1994.

12.) Schwizer W., Fraser R., Borovicka J., Crelier G., Boesiger P., Fried M. Measurement of Gastric Emptying and Gastric Motility by Magnetic Resonance Imaging (MRI). Digestive Diseases and Sciences 39(12), 101S-103S, 1994. 